\title{
Mundos virtuales como canal de comunicación entre escuelas y museos
}

\author{
Luis Antonio HeRnÁNDEZ IBÁÑEZ \\ VideaLAB. Universidad de la Coruña \\ lhernandez@udc.es \\ Viviana BARNECHE NAYA \\ VideaLAB. Universidad de la Coruña. \\ viviana.barneche@udc.es \\ Rocío MiHuRa LÓPEZ \\ VideaLAB. Universidad de la Coruña \\ mihura@udc.es
}

Recibido: 24/07/2012

Aceptado: 29/10/2012

\section{Resumen}

Este artículo describe los resultados sobre el estudio de la experiencia del usuario de dos colegios dentro de un espacio virtual educativo flexible que conjuga escuela y museo. Este espacio educativo integral incluye no solo la exploración de áreas expositivas, sino que abarca desde charlas telepresenciales por parte del personal del museo, simulaciones, virtual quests a trabajos escolares dentro de un entorno virtual multiusuario basado en OpenSim, accesible simultáneamente desde las diferentes instituciones implicadas en el experimento.

Palabras clave: Mundos Virtuales, Metaversos, Experiencia de usuario, Virtual learning, Código abierto.

\section{Virtual worlds as a channel of communication between schools and museums}

\begin{abstract}
This paper describes the results about the study of the user experience of two schools within a flexible virtual space that connects school and museum. This integrated educational space is not only uses for the exploration of exhibition areas but also includes telepresence talks on the part of museum personnel, simulations, educational work in the form of virtual quests, all within a multi-user virtual environment based on OpenSim and simultaneously accessible from the different institutions involved in the experiment.

Keywords: Virtual Worlds, Metaverses, User Experience, Virtual learning, Open source.

\section{Referencia normalizada}

HERNÁNDEZ IBÁÑEZ, Luis Antonio; BARNECHE NAYA, Viviana y MIHURA LÓPEZ, Rocío (2012): "Mundos virtuales como canal de comunicación entre escuelas y museos". Estudios sobre el mensaje periodístico. Vol. 18, núm. especial noviembre, págs.: 509-518. Madrid, Servicio de Publicaciones de la Universidad Complutense.
\end{abstract}

Sumario: 1. Introducción. 2. Metodología. 3. Desarrollo; 3.1. Temática; 3.2. Diseño de la experiencia; 3.3. Aspectos técnicos; 3.4. Contenidos; 3.4.1. Role Playing; 3.4.2. Asistencia a una charla; 3.4.3. Exploración libre; 3.4.4. Exploración asistida; 3.4.5. Simulación; 3.4.6. Virtual Quest; 3.5. Escenarios. 4. Resultados; 4.1. Usabilidad; 4.2. Presencia; 4.3. Aprendizaje; 4.4. Satisfacción de los alumnos; 4.5. Impresión de los responsables; 4.6. Aspectos para revisar. 5. Conclusiones. 6. Referencias bibliográficas. 


\section{Introducción}

Entre las diferentes tecnologías utilizadas en el ámbito del Aprendizaje Colaborativo Asistido por Ordenador (o CSCL por sus siglas en inglés), los mundos virtuales han estado en los últimos años recibiendo especial atención por cuanto constituyen un medio de inmensas potencialidades en el ámbito de la teleformación.

La tridimensionalidad del espacio que aloja las actividades llevadas a cabo en ellos, así como la representación de los usuarios en forma de avatar, aportan una sensación de inmersión en un espacio común y un grado de presencia, de consciencia de la compañía de otros estudiantes y de los profesores, características que resultan incitadoras al aprendizaje como se plasma en diversos estudios (Duncan, 2012; Schroeder et al, 2006).

Son muchos los ejemplos desarrollados por instituciones de educación superior en el diseño de actividades formativas en mundos virtuales (Wankel \& Kingsley2009; Dalgarno et al, 2010), tales como el entrenamiento de estudiantes de Medicina en habilidades de entrevista para diagnosis o la representación de juicios virtuales para estudiantes de Derecho.

La mayor parte de los estudios acerca del rendimiento comparativo entre estos entornos virtuales colaborativos y sus situaciones equivalentes del mundo real han sido llevados a cabo para ese rango etario, especialmente en plataformas MUVE (MultiUser Virtual Environment) como Second Life, que cuentan con el requisito de edad mínima para el acceso a las mismas, lo que impide su uso por parte de otros colectivos, tales como colegios de educación primaria.

Esta exigencia no existe si se usan plataformas MUVE de código abierto como OpenSim, con las que es posible generar entornos virtuales privados de aprendizaje.

Los mundos virtuales para niños crecen día a día, y sin embargo, hay pocos estudios sobre el uso que hacen los niños de estos mundos (Marsh, 2010). Si son pocos los casos documentados sobre experiencia de usuario en VWs aplicadas a la educación superior (Savin-Baden et Al, 2010) son más escasas aún las centradas específicamente en niños (Barab et Al, 2007; Arhippainen et al, 2011). No obstante, existe una cantidad relevante de blogs y wikis con impresiones y hojas de ruta de experiencias como Suffern Middle School (Sheehy 2008), DigiTeen Project (Lindsay \& Davis, 2008), o CANVAS (Robertson, 2009) (Children's Art at the National Virtual Arena of Scotland), el cual es parte del programa educativo GLOW, la comunidad online de escuelas escocesas.

El diseño de una experiencia formativa integral que relacione escuelas con otras instituciones como los museos dentro de un VWs permite obtener nuevos enfoques de la experiencia del usuario- niño y evaluar la eficiencia de los mismos en los procesos de aprendizaje. En este contexto el museo deja de ser simplemente un espacio de exposiciones y simulaciones dentro del espacio virtual para pasar a formar parte activa del espacio de formación del colegio donde maestros y científicos diseñan, coordinan, e imparten telepresencialmente las diferentes actividades.

A partir de lo anteriormente expuesto, este artículo busca analizar la experiencia de usuario (UX) de una actividad escolar grupal, exponiendo un ejemplo de diseño y desarrollo de una actividad escolar especial, la visita a un museo, a través del uso de un entorno virtual multiusuario basado en OpenSim. 


\section{Metodología}

La experiencia se plantea como objetivo general comprobar la viabilidad de los mundos virtuales como plataforma para llevar a cabo actividades escolares que relacionen colegios y museos, más allá de la simple exploración de exposiciones virtuales, sino implicando al personal de los museos en el desarrollo de actividades formativas del mismo alcance de las que se desarrollarían en la visita de una clase de escolares a un museo en el mundo real.

Tras la experiencia, se realizaría una evaluación de los distintos aspectos relacionados tanto con la funcionalidad como con la calidad de la misma, tanto a nivel de presencia como de enseñanza/aprendizaje, y tanto para el colegio como para el museo. La misma se llevaría a cabo mediante entrevistas a los responsables de los centros y del museo, y mediante encuestas a los alumnos.

Con los datos obtenidos se analizarán múltiples aspectos relacionados con la experiencia de usuario centrada en niños en edad escolar en cuanto a la percepción del espacio y el grupo, y la usabilidad del entorno, considerando las condiciones de exigencia como son las de una clase real para usuarios no familiarizados con el uso de este entorno, comparando distintas alternativas de uso en dos escenarios diferentes.

Esta experiencia educativa involucró a tres clases de educación primaria de $6^{\circ}$ curso - 11-12 años - de dos colegios distintos de A Coruña, ensayándose en cada caso diferentes variantes de la experiencia. La dos primeras, llamémosla Clase A y Clase B corresponden a un colegio privado/concertado, las Esclavas del Sagrado Corazón de Jesús. La tercera, que llamaremos Clase C corresponde con un colegio público, el Sanjurjo de Carricarte.

Como colaboradores se contó con los Museos Científicos Coruñeses (mc2), que aportó a su personal técnico y docente para impartir una clase de Ciencias telepresencialmente, y guiar la visita del grupo de alumnos/avatares a través de las instalaciones virtuales visitando un planetario virtual y una pirámide maya.

Posteriormente y dentro del mundo virtual, los estudiantes realizaron tareas escolares relacionadas durante el transcurso de la propia actividad, a través de un juego de exploración, investigando determinados objetos expuestos y rellenando un cuestionario de preguntas sobre lo aprendido.

Finalmente, los niños que tomaron parte en la misma rellenaron una encuesta cuyos resultados permitieron extraer las diversas conclusiones que cierran este trabajo, abriendo nuevas líneas de investigación.

\section{Desarrollo}

\subsection{Temática}

El tema de la experiencia, que fue acordado conjuntamente por los equipos directores de los colegios y los responsables pedagógicos del museo fue el Sistema Solar, englobando tanto la unidad didáctica correspondiente y sus tareas asociadas, como el aporte extraordinario dado por el museo, que consistiría en una charla sobre la astronomía de los mayas, su profecía del fin del mundo en 2012 y el auténtico final del Sistema Solar dentro de 4.000 millones de años. 


\subsection{Diseño de la experiencia}

El equipo realizador del trabajo ya tenía experiencia en el desarrollo de actividades de formación en mundos virtuales, aunque estas fueron en su mayoría dirigidas a estudiantes adultos e impartidos por profesores familiarizados con educación telepresencial en mundos virtuales. (Barneche y Hernández 2008)

Esta primera incursión dentro de los metaversos brindó el "know-how" necesario para posteriormente investigar acerca de cuál es la tecnología más adecuada para trabajar en este tipo de proyectos. Sin embargo, esta experiencia sería totalmente distinta en todos los aspectos de los casos anteriores. Los alumnos serían niños, que accederían por primera vez a un mundo de estas características.

El profesor del museo recibió una pequeña formación previa en el manejo del sistema de cara a la preparación de su intervención y el ensayo de la misma, así como para asistir al equipo de diseño en la construcción de los contenidos desde dentro del propio mundo.

\subsection{Aspectos técnicos}

El uso de software de código abierto resulta esencial en este tipo de proyectos tecnológicos para evitar adaptar las necesidades del proyecto a las limitaciones de un software propietario. Para proyecto Escola se utilizó como plataforma de soporte a OpenSim, por diversos motivos:

- Siendo una plataforma de código abierto, puede establecerse una red de servicio privada con acceso perfectamente controlado.

- No hay más limitaciones en cuanto a cantidad y tipo de contenidos que pueden utilizarse que las derivadas de los límites técnicos de la misma.

- No hay requisitos de edad mínima de acceso. Ello descartó el uso de otras soluciones comerciales más robustas, pero de acceso restringido a niños.

- Facilidad para la creación de entornos tridimensionales in-world, Capacidad de programación mediante scripting. Soporte multimedia. VoIP

Se puso en funcionamiento un servidor OpenSim que alojó una isla virtual en la que desarrollar la experiencia, accesible tanto por las escuelas como por el museo, creándose una red (grid) privada para la ocasión, y cuentas de usuario para los organizadores, así como cuentas de usuario con nombres genéricos para los estudiantes.

Para el acceso a la misma, se personalizó el visor de código abierto Imprudence por su sencillez y robustez, que fue instalado en los equipos del propio centro educativo en las clases A y B y en ordenadores portátiles del equipo responsable de la experiencia en la Clase $\mathrm{C}$.

\subsection{Contenidos}

La experiencia, cuya duración debería ser de en torno a dos horas, tal como sucedería en una visita a museo normal, podría incluir, a pesar de su corta duración, actividades de distintos tipos de entre las categorizadas en la literatura existente (Duncan, 2012: 953) como soporte para la enseñanza y la educación en mundos virtuales: 


\subsubsection{Role Playing}

Los avatares que representarían a los participantes debería reflejar el rol de los mismos en el mundo real. Para ello se crearon avatares con apariencia de niños y niñas de doce años, y avatares de adulto para el profesor y el formador del museo, que vestiría además con gafas y su bata de científico de laboratorio.

Los alumnos dedicarían una parte de la experiencia a personalizar su apariencia en el mundo virtual, para lo que se prepararon doce patrones de avatar de niña y ocho de niño sobre los que personalizaron su apariencia.

\subsubsection{Asistencia a una charla}

El formador del museo impartió una clase en el aula preparada para tal fin en el mundo virtual. Para ello contó dentro del mismo con una pantalla, en la que expuso un vídeo sobre la formación del sistema solar y algunas imágenes de apoyo.

Por la temática de su exposición, utilizó también un modelo tridimensional de la estela maya utilizada para difundir el rumor del fin del mundo para finales del 2012, explicando la falta de fundamento de esa teoría, sin desmerecer los conocimientos de astronomía de esa civilización.

\subsubsection{Exploración libre}

El mundo virtual contenía una réplica de la pirámide maya de Kukulcán en Chichén Itzá. La pirámide no solo sirvió para hablar sobre la arquitectura del mundo maya sino también para relacionar su perfecta orientación respecto a los puntos cardinales con sus conocimientos en astronomía, que se materializó ubicando la hora del mundo virtual en el atardecer, de forma que desde su cima los niños pudieron contemplar la puesta de sol por el Ocaso, perfectamente alineada con la escalinata principal del templo.

\subsubsection{Exploración asistida}

Los alumnos pudieron viajar con el científico explorando los planetas para dirigirse finalmente a Saturno, ya a su luna Titán, mientras se explicaba su candidatura a ser un futuro hogar para la humanidad en ese tiempo del futuro distante.

\subsubsection{Simulación}

El mundo no acabará en diciembre, (o al menos no en diciembre de este año), aunque es cierto que la ciencia dice que terminará siendo absorbido por el Sol cuando éste se convierta en una estrella gigante roja, dentro de 4.000 millones de años.

Para ilustrar esto, se modeló un planetario virtual, poniendo el mundo en modo nocturno, en el que el científico pudo moverse entre los planetas, hablando de ellos y del fenómeno de crecimiento de la estrella. Este fenómeno fue simulado con el aumento en tamaño del Sol hasta engullir los planetas interiores del Sistema Solar, incluida la Tierra llegando hasta la proximidad de Marte.

\subsubsection{Virtual Quest}

El mundo alojó una sala en la que se expusieron modelos interactivos de los distintos objetos del Sistema Solar. El Sol, los planetas, la Luna y una réplica de la sonda espacial Voyager II. 
Se planteó a los niños la posibilidad de llevar a cabo una búsqueda de pistas. A través de la interacción con los modelos de los planetas, obtendrían notas con datos sobre los mismos.

Se les facilitó un cuestionario en papel real (que serían sus tareas) con preguntas sobre el Sistema Solar. Las respuestas eran numéricas, de forma que extrayendo alguna de sus cifras, se formaba un código secreto que podría introducirse en la sonda espacial. Si el código era correcto, la nave les daría un premio, consistente en una imagen del disco de oro que portaba en su interior, con un mensaje dirigido a civilizaciones de otros mundos.

Tras completar sus tareas, y obtener su premio, los niños salieron del mundo virtual.

\subsection{Escenarios}

La experiencia se planteó en dos escenarios distintos debido a las distintas condiciones de acceso a Internet de los colegios públicos y privados:

- Experiencia inmersiva: La actividad en las Clases A y B fue en su totalidad llevada a cabo en el mundo virtual.

- Blending Learning: La existencia de un cortafuegos corporativo en el sistema de enseñanza pública no permitía utilizar los puertos necesarios lo que imposibilitaba el acceso al mundo virtual desde el aula de ordenadores de la Clase C.

Este inconveniente era previamente conocido, por lo que se decidió utilizar en este escenario un planteamiento mixto de clase telepresencial y actividad inmersiva.

La comparación de los resultados obtenidos en los distintos escenarios en la búsqueda de idénticos objetivos con actividades de diferentes tipos arrojó interesantes conclusiones.

\section{Resultados}

Para la evaluación de la experiencia se recopiló información a través de una encuesta que los niños realizaron en los días posteriores.

En ella se plantearon preguntas relativas a tres aspectos: usabilidad, presencia y aprendizaje, así como otras de percepción comparada de la actividad en relación con otras actividades realizadas en vivo y una última de satisfacción general.

Las preguntas se realizaron en un lenguaje amigable para los niños. Todas las preguntas de la encuesta podían tomar valores cualitativos entre 1 (peor/poco/mal) y 5 (mejor/mucho/bien).

\subsection{Usabilidad}

El manejo del interfaz y el movimiento dentro del mundo como caminar, volar, enfocar la cámara, etc. obtuvieron notas muy altas (4.28/4.41).

La observación de los monitores corroboró una sorprendente facilidad de niños en su primera experiencia con este tipo de plataformas formativas para utilizar un interfaz diseñado originalmente para personas de más edad sin el más mínimo problema.

La personalización del avatar, siendo un proceso algo más complejo tampoco planteó grandes dificultades. (3.74/3.94). 


\begin{tabular}{|c|c|c|c|}
\hline Preguntas & Clase & Valoración (1-5) & $\sigma$ \\
\hline \multirow{2}{*}{ ¿Te resultó fácil la exploración del Mundo Virtual (moverte, volar, chatear)? } & $\mathrm{AB}$ & 4.28 & 1.04 \\
\hline & $\mathrm{C}$ & 4.41 & 0.91 \\
\hline \multirow{2}{*}{ ¿Cómo te ha resultado modificar la apariencia de tu avatar? } & $\mathrm{AB}$ & 3.74 & 1.25 \\
\hline & $\mathrm{C}$ & 3.94 & 1.21 \\
\hline \multirow{2}{*}{$\begin{array}{l}\text { ¿Podias seguir fácilmente la conferencia sobre las profecias Mayas y el fin del } \\
\text { Sistema Solar? }\end{array}$} & $\mathrm{AB}$ & 3.60 & 0.81 \\
\hline & $\mathrm{C}$ & 4.41 & 0.77 \\
\hline \multirow{2}{*}{$\begin{array}{l}\text { ¿Sentías al profesor que estaba dándote clases en el Mundo Virtual igual que lo } \\
\text { harias con un profesor en el aula real? }\end{array}$} & $\mathrm{AB}$ & 3.92 & 1.15 \\
\hline & $\mathrm{C}$ & 4.29 & 1.13 \\
\hline \multirow{2}{*}{$\begin{array}{l}\text { Con respecto a tus compañeros ¿Tenias la sensación de estar juntos dentro del } \\
\text { Mundo Virtual? }\end{array}$} & $\mathrm{AB}$ & 4.13 & 0.90 \\
\hline & $\mathrm{C}$ & 4.24 & 0.55 \\
\hline \multirow{2}{*}{ ¿Cómo te resultó la actividad final del cuestionario sobre el Sistema Solar? } & $\mathrm{AB}$ & 3.96 & 1.25 \\
\hline & $\mathrm{C}$ & 4.18 & 0.98 \\
\hline
\end{tabular}

Si tuvieses que comparar con otras actividades, el Mundo Virtual te resultó una experiencia parecida a ....

\begin{tabular}{|c|c|c|c|}
\hline \multirow{2}{*}{ Ir a una clase } & $\mathrm{AB}$ & 2.57 & 1.17 \\
\hline & $\mathrm{C}$ & 2.94 & 1.00 \\
\hline \multirow{2}{*}{ Ir de visita al planetario } & $\mathrm{AB}$ & 3.93 & 0.95 \\
\hline & $\mathrm{C}$ & 4.12 & 1.02 \\
\hline \multirow{2}{*}{ Ir de excursión con la clase } & $\mathrm{AB}$ & 3.21 & 1.28 \\
\hline & $\mathrm{C}$ & 3.18 & 1.42 \\
\hline \multirow{2}{*}{ Visitar un museo interactivo } & $\mathrm{AB}$ & 3.86 & 1.15 \\
\hline & $\mathrm{C}$ & 4.29 & 1.02 \\
\hline \multirow{2}{*}{ Ver un video o una película } & $\mathrm{AB}$ & 3.03 & 1.42 \\
\hline & $\mathrm{C}$ & 3.53 & 1.29 \\
\hline \multirow{2}{*}{ Jugar un videojuego } & $\mathrm{AB}$ & 3.98 & 1.19 \\
\hline & $\mathrm{C}$ & 4.59 & 0.6 \\
\hline \multirow{2}{*}{ ¿Te gustaria repetir esta experiencia en otra asignatura? } & $\mathrm{AB}$ & 100 & 0 \\
\hline & $\mathrm{C}$ & 100 & 0 \\
\hline \multirow{2}{*}{ ¿Consideras que aprendiste cosas nuevas tras tu paso por el mundo virtual? } & $\mathrm{AB}$ & 4.24 & 0.93 \\
\hline & $\mathrm{C}$ & 4.71 & 0.57 \\
\hline \multirow{2}{*}{ ¿Esta primera exploración dentro del Mundo Virtual te ha resultado? } & $\mathrm{AB}$ & 4.74 & 0.52 \\
\hline & C & 4.63 & 0.48 \\
\hline
\end{tabular}

Tabla 1. Resultados de la encuesta planteada a los estudiantes de ambos colegios. Fuente: elaboración propia 


\subsection{Presencia}

La sensación de presencia en el mundo virtual en compañía del grupo fue considerada por los niños como alta/muy alta (4.12/4.24), así como el sentido de presencia del profesor del museo comparado con un profesor de una clase real (3.92/4.29).

Aquí cabe resaltar el dato de que la sensación de presencia del profesor remoto en la clase fue mayor para los alumnos que vieron la charla en la pantalla del aula (Clase C) que para los que lo vieron desde dentro del mundo (clases A y B).

Entendemos que ello fue debido a varias razones: Por una parte, el hecho de estar sentados, mirando al conferenciante en la pantalla sin distracciones posibles aumentó la concentración en la charla (como además reafirmaría una pregunta posterior sobre la comprensión de la misma) en comparación a las clases A-B en que los niños tenían más posibilidades de distraerse haciendo otras cosas dentro del mundo; comportamiento similar al que sucede en las charlas reales en clase cuando nadie mira.

Por otra parte, la proyección del avatar en pantalla grande, aproxima su aspecto al tamaño real de un profesor. No obstante, las clases A y B también reportaron un gran nivel de presencia del profesor.

\subsection{Aprendizaje}

Relacionado con lo anterior, los alumnos de la Clase $\mathrm{C}$ respondieron más positivamente a la pregunta relativa al grado de comprensión de la charla. (4.41) que los de las clases A y B (3.60). Ello parece indicar que el uso de mundo virtual como medio para la formación telepresencial, sin necesidad de inmersión por parte de los alumnos tiene una gran efectividad, tanto en la sensación de presencia como en el aprendizaje. $\mathrm{Su}$ uso en esta modalidad no es por tanto desdeñable.

Respecto al cuestionario de preguntas, respondido a través del Virtual Quest, la facilidad en su resolución fue calificada con medias altas (3.96/4.18). En opinión de los profesores del centro educativo, la dificultad del test podría considerarse alta para ese rango de edad, por lo que parece que la motivación, el medio y el mecanismo para obtener las respuestas pudieron hacer que los alumnos no percibiesen la dificultad de la tarea que estaban llevando a cabo.

\subsection{Satisfacción de los alumnos}

De cara a medir la satisfacción de los estudiantes ante este tipo de experiencia formativa, se plantearon dos preguntas: Deseo de repetir con otra asignatura distinta, que fue abrumadoramente afirmativo (5/5) con desviación típica 0 , e impresión general de la experiencia, que calificaron de muy buena (4.74/4.63) con desviación típica en torno a 0.5 .

\subsection{Impresión de los responsables}

Ambas direcciones de las dos escuelas manifestaron un alto grado de satisfacción con la experiencia, nueva para ellos, ofreciéndose para ser repetida en el futuro con más grupos. Por lo que respecta a los responsables del museo, resaltaron el potencial de esta tecnología para llegar con mucha facilidad a uno de sus públicos objetivo, los colegios, ofreciéndose para estudiarla en más profundidad. 


\subsection{Aspectos para revisar}

Aunque el resultado general de la experiencia fue altamente satisfactorio, se identificaron algunos aspectos a corregir.

Los alumnos mostraron diferente grado de concentración a lo largo de las distintas actividades, tendiendo a dispersarse cuando el objetivo no estaba claramente señalado como en el caso de la excursión a la pirámide, lo que implicaba cierto esfuerzo de organización para reagruparlos.

La posibilidad de experimentar con funciones del visor sin papel específico en el experimento, o con un uso que debía ser restringido a solo una parte de la experiencia provocó que en algunos casos se distrajeran haciendo otras actividades como chatear. También se detectaron varios casos de alumnos que se distraían modificando su apariencia cuando deberían estar atendiendo al docente.

No obstante, los profesores del centro manifestaron que tal tipo de actitudes era común en una visita a un museo real. En futuras experiencias se procurará mejorar el diseño de la experiencia en estos aspectos y utilizar más las posibilidades del sistema para centrar mejor a los alumnos en la actividad que deben realizar y evitar distracciones.

\section{Conclusiones}

El uso del mundo virtual para la educación de niños en edad escolar permite que los mismos adquieran conocimientos de forma natural y con la sensación más de un juego que de una clase esforzada debido al carácter lúdico de los medios y herramientas utilizadas durante el aprendizaje

Este medio puede ser utilizado para emular no solo la experiencia de una clase telepresencial, sino el conjunto de actividades presente en una actividad grupal extraescolar como la visita a un museo. En este artículo se verifica la viabilidad tanto de la metodología como la logística necesarios para llevarlo a cabo.

Es destacable la naturalidad con que los alumnos jóvenes perciben la presencia de profesores remotos y se involucran en este tipo de experiencia. La utilización de varios formatos como la simple proyección del avatar del docente distante en el aula virtual en una pantalla real constituye una posibilidad sencilla y efectiva para permitir la impartición de charlas remotas, incluso a varias clases simultáneamente.

Entre las líneas futuras de investigación en este campo, se está trabajando en la experimentación con actividades de encuentro que involucren la colaboración entre varios grupos escolares distantes, y el planteamiento de actividades educativas coordinadas con los museos participantes para grupos de edad inferior.

\section{Referencias bibliográficas}

ARHIPPAINEN Leena, PAKANEN Minna, HICKEY Seamus, MATTILA Pasi (2011): User Experiences of 3D Virtual Learning Environment. Proceedings of the 15th International Academic MindTrek Conference: Envisioning Future Media Environments, pp:222-227. DOI= http://dl.acm.org/citation.cfm?doid=2181037. 2181075

BARAB, Sasha; DODGE, Tyler; THOMAS, Michael; JACKSON, Craig y TUZUN Hakan (2007): "Our Designs and the Social Agendas They Carry". The Journal of the Learning Sciences, 16, pp: 263-305. 
BARNECHE, Viviana; HERNÁNDEZ, Luis (2008): Ciberarquitectura Educativa. La experiencia de Isla Videa en Second Life. XII Congreso Iberoamericano de Gráfica Digital, SIGRADI 2008. Ed Cujae, 2008.

DALGARNO, Barney; LEE, Mark J. W. (2010): "What are the learning affordances of 3-D virtual environments?" British Journal of Educational Technology 41, 1 (2010), 10-32.

DUNCAN, Isabel; MILLER, Alan; JIANG, Shangyi (2012): "Taxonomy of virtual worlds usage in education". British Journal of Educational Technology, Vol. 43, pp 949-964. DOI:10.1111/j.1467-8535.2011.01263.x

LINDSAY Julie, DAVIS Vicki (2008): “DigiTeen Project”. En DigiTeen: http://digiteen.ning. com/

MARSH Jackie (2010): "Young children's play in online virtual worlds". Journal of Early Childhood Research, February 2010; vol. 8, 1, pp. 23-39.

ROBERTSON, Derek (2009): “CANVAS: Scotland's first schools based virtual world for learning". En Consolarium: http://tsblogs. org.uk/consolarium/.

SAVIN-BADEN Maggi, GOURLAY Lesley, TOMBS Cathy, STEILS Nicole, TOMBS Gemma and MAWER Matt (2010): "Situating pedagogies, positions and practices in immersive virtual worlds". Virtual Worlds and Education, Volume 52, Issue 2, 2010. Special Issue:, pp.123-133. DOI:10.1080/00131881.2010.482732

SCHROEDER, Ralph; HELDAL, Ilona; TROMP, Jolanda (2006): "The Usability of Collaborative Virtual Environments and Methods for the Analysis of Interaction". Presence. MIT Press Journal. Dec. 2006, vol. 15, No. 6, 655-667. DOI:10.1162 /pres.15.6.655.

SHEEHY, Peggy (2008): "Suffern Middle School in Virtual Worlds". En: http://ramapoislands. edublogs. org/.

WANKEL Charles, KINGSLEY Jan (2009): Higher Education in Virtual World. Teaching and learning in Second Life. Esmerald Group Publishing Limited. 


\section{Luis Antonio HERNÁNDEZ IBÁÑEZ}

VideaLAB. Universidad de la Coruña.

lhernandez@udc.es

Doctor Arquitecto, Universidad de A Coruña, España. Director del Grupo de Visualización Avanzada en Arquitectura, Ingeniería Civil y Urbanismo - VideaLAB, Director del Máster en Creación y Comunicación Digital. (1998-2009). Profesor Titular en la Escuela Técnica Superior de Ingenieros de Caminos, Canales y Puertos; y de la Facultad de Ciencias de la Comunicación de la Universidad de A Coruña. Autor de múltiples publicaciones en el ámbito de la visualización avanzada, la ciberarquitectura y la recreación del Patrimonio. Sus líneas de investigación en enmarcan en el campo de la creación digital, la simulación por computador, las Interfaces Naturales, los Mundos Virtuales 3D y la Realidad Virtual.

\section{Viviana BARNECHE NAYA}

VideaLAB. Universidad de la Coruña.

viviana.barneche@udc.es

Arquitecta, Universidad de la República, Uruguay. PhD candidate, en etapa de Tesis, Universidad de A Coruña. Docente de Taller de Anteproyectos, Facultad de Arquitectura, UdelaR (2001-2011). Docente dentro del Máster en Creación y Comunicación Digital, Universidad de A Coruña (2008-2009). Profesora interina, Universidad de A Coruña (2011). Sus líneas de investigación se enmarcan en los campos de la creación digital interactiva, el diseño de ciberarquitectura y contenidos dentro de los Metaversos, la recreación patrimonial, la simulación por computador y las Interfaces Naturales.

\section{Rocío MIHURA LÓPEZ}

VideaLAB. Universidad de la Coruña.

mihura@udc.es

Licenciada en Bellas Artes por la Universidade de Vigo. Máster en Creación y Comunicación Digital por la Universidade da Coruña. PhD candidate, en etapa de Tesis, Universidad de A Coruña. Profesora en la Facultade de Ciencias da Comunicación de la Universidade da Coruña. Sus líneas de investigación abarcan los campos de la creación digital interactiva en el ámbito de la museística y los contenidos audiovisuales dentro de los Museos. 